\title{
Critical phenomena of the hard-sphere lattice gas on the simple cubic lattice
}

\author{
Atsushi Yamagata \\ Department of Physics, Tokyo Institute of Technology, Oh-okayama, Meguro- \\ ku, Tokyo 152, Japan \\ and \\ Department of Physics, Tokyo Metropolitan University, Minami-ohsawa 1-1, \\ Hachioji-shi, Tokyo 192-03, Japan
}

Running title Critical phenomena of hard-sphere lattice gas

Keywords Hard-sphere lattice gas, Critical phenomena, Monte Carlo method PACS classification codes 02.70.Lq, 64.60.Cn, 68.35.Rh

\begin{abstract}
We study the critical phenomena of the hard-sphere lattice gas on the simple cubic lattice with nearest neighbour exclusion by the Monte Carlo method. We get the critical exponents, $\beta / \nu=0.313(9)$ and $\gamma / \nu=2.37(2)$, where $\beta$ is the critical exponent for the staggered density, $\gamma$ for the staggered compressibility, and $\nu$ for the correlation length.
\end{abstract}




\section{Introduction}

Atoms of a hard-sphere lattice gas occupy sites of a lattice and interact with infinite repulsion of nearest neighbour pairs. The grand partition function is

$$
\Xi_{V}(z)=\sum_{N} z^{N} Z_{V}(N)
$$

where $z$ is an activity and $Z_{V}(N)$ is the number of configurations in which there are $N$ atoms in the lattice of $V$ sites. At $z=+\infty$ a ground state configuration is that the atoms occupy all the sites of one sublattice and the other is vacant. There is no atom at $z=0$. A continuous phase transition occurs at a critical activity. There are many studies of the system by various methods: series expansions [1, 2, 3, 4, 5], finite-size scaling and transfer matrix [6], Bethe and ring approximations [7], transfer matrix [8, 9, 10], corner transfer matrix and series expansions [11], exact calculations [12], and Monte Carlo simulations [13, 14.

The critical activity is obtained on various lattice. The square lattice: 3.80(2) [4], 3.80(4) [9], 3.7966(3) [10], 3.7962(1) [11], and 3.796255174(3) [15]. The triangular lattice: 11.12(11) [9], 11.05(15) [5], and $\frac{1}{2}(11+5 \sqrt{5})=$ $11.090 \ldots$ [12]. The simple cubic lattice: 1.09(7) [5] and 1.0588(3) [14]. The body-centred cubic lattice: $0.77(5)$ [5] and 0.73(2) [16, 17]. The critical exponents on the square lattice are as follows. The thermal exponent, $y_{t}$, is $1.000000(1)$, the magnetic exponent, $y_{h}$, is $1.875000(1)$ [ [6], $\beta / \nu=0.125(5)$, and $\gamma / \nu=1.74(2)$ 13 where $\beta, \gamma$, and $\nu$ are the critical exponents for the order parameter, the staggered compressibility, and the correlation length, respectively. There are exact values on the triangular lattice [12, 18]: $\alpha=1 / 3$ for the compressibility, $\beta=1 / 9, \mu=5 / 6$ for the interfacial tension, and $\nu=5 / 6$. The value, $0.61071(2)$ [19], of the fourth-order cumulant [20] of the order parameter on the square lattice agrees with those of the Ising model, $0.611(1)$ [21], 0.6103(7) [22], 0.611(1), and 0.610690(1) [19]. These results

support that the hard-sphere lattice gas on the square lattice belongs to the universality class of the two-dimensional Ising model and that on the triangular lattice does to one of the three-state Potts model in two dimensions [23]. There is no result of critical exponents in three-dimensional hard-sphere lattice gas.

We carry out Monte Carlo simulations of the hard-sphere lattice gas on the simple cubic lattice. Using the finite-size scaling, we estimate the critical 
exponents. In the next section we define physical quantities measured. In section 3 we describe procedure of analyses and present Monte Carlo results. A summary is given in section 1 .

\section{Monte Carlo simulations}

We use the Metropolis Monte Carlo technique [24, 25] to simulate the hardsphere lattice gas (11) on the simple cubic lattice of $V$ sites, where $V=L \times$ $L \times L(L=2 \times n, n=2,3, \ldots, 30)$, under fully periodic boundary conditions. According to Meirovitch [13, we adopt the grand canonical ensemble. The algorithm is described in the references [13, 14.

We start each simulation with a ground state configuration at the critical activity, $z_{\mathrm{c}}=1.0588$ [14]. The pseudorandom numbers are generated by the Tausworthe method [26, 27]. We measure physical quantities over $1.2 \times 10^{6}$ Monte Carlo steps per site (MCS/site) for $L \geq 44$ and $10^{6} \mathrm{MCS} /$ site for $L \leq 42$ after discarding $5 \times 10^{4} \mathrm{MCS} /$ site to attain equilibrium. We have checked that simulations from the ground state configuration and no atom one gave consistent results. Each run is divided into $M$ blocks. Let us the average of a physical quantity, $O$, in each block $\langle O\rangle_{i} ; i=1,2, \ldots, M$. The expectation value is

$$
\overline{\langle O\rangle}=\frac{1}{M} \sum_{i=1}^{M}\langle O\rangle_{i} .
$$

The standard deviation is

$$
\Delta\langle O\rangle=\left(\overline{\langle O\rangle^{2}}-\overline{\langle O\rangle}^{2}\right)^{1 / 2} / \sqrt{M-1} .
$$

We have taken $M=12$ for $L \geq 44$ and $M=10$ for $L \leq 42$.

Let us define an order parameter by

$$
R=2\left(N_{\mathrm{A}}-N_{\mathrm{B}}\right) / V
$$

where $N_{\mathrm{A}}\left(N_{\mathrm{B}}\right)$ is the number of the atoms in the $\mathrm{A}(\mathrm{B})$-sublattice and $N=N_{\mathrm{A}}+N_{\mathrm{B}}$. We measure the staggered densities,

$$
m^{\dagger}=\overline{\langle|R|\rangle}
$$

and

$$
m^{\dagger \prime}=\overline{\left\langle R^{2}\right\rangle^{1 / 2}}
$$


the staggered compressibilities,

$$
\chi^{\dagger}=V \overline{\left(\left\langle R^{2}\right\rangle-\langle|R|\rangle^{2}\right)} / 4
$$

and

$$
\chi^{\dagger \prime}=V \overline{\left\langle R^{2}\right\rangle} / 4
$$

and the fourth-order cumulant of $R$ [20],

$$
U=1-\frac{1}{3} \overline{\left\langle R^{4}\right\rangle /\left\langle R^{2}\right\rangle^{2}}
$$

\section{Monte Carlo results}

We estimate a critical exponent and an amplitude by using the finite-size scaling [28, 29, 30]. For a physical quantitiy, $O$, we use the nonlinear chisquare fitting [31] with a function of $L$,

$$
O(L)=A L^{p}
$$

where $p$ and $A$ are fitting parameters. $p=-\beta / \nu$ when $O=m^{\dagger}$ or $m^{\dagger \prime}$. $p=\gamma / \nu$ when $O=\chi^{\dagger}$ or $\chi^{\dagger \prime}$. We calculate the chi-square per degrees of freedom, $\chi^{2} / \mathrm{DOF}$, and the goodness of fit, $Q$, [31 for the data set of the sizes $L=L_{\min }, L_{\min }+2, \ldots, L_{\max }-2$, and $L_{\max }$. The values of $L_{\min }$ and $L_{\max }$ are selected so that the difference between $\chi^{2} / \mathrm{DOF}$ and 1 is the smallest.

We get the results as follows. For $m^{\dagger}$ defined by (2), $\beta / \nu=0.28(1), A$ $=0.39(2), \chi^{2} / \mathrm{DOF}=1.21$, and $Q=0.27$. For $m^{\dagger \prime}$ defined by (3), $\beta / \nu=$ $0.313(9), A=0.48(2), \chi^{2} / \mathrm{DOF}=1.22$, and $Q=0.26$. For $\chi^{\dagger}$ defined by (田), $\gamma / \nu=1.96(2), A=0.040(3), \chi^{2} / \mathrm{DOF}=0.98$, and $Q=0.47$. For $\chi^{\dagger \prime}$ defined by (5), $\gamma / \nu=2.37(2), A=0.058(4), \chi^{2} / \mathrm{DOF}=1.21$, and $Q=0.28$. $L_{\min }=36$ and $L_{\max }=60$ for $m^{\dagger}, m^{\dagger \prime}$, and $\chi^{\dagger \prime} . L_{\min }=30$ and $L_{\max }=54$ for $\chi^{\dagger}$.

The values of the critical exponents of $m^{\dagger \prime}$ and $\chi^{\dagger \prime}$ satisfy the scaling relation, $2 \beta+\gamma=d \nu$ where $d$ is the dimensions, within errors. Thus we adopt them as the reliable estimates. Figure 1 shows the size dependence of $m^{\dagger \prime}$ at $z=z_{\mathrm{c}}$. The solid line indicates $0.48 L^{-0.313}$. Figure 2 shows the size dependence of $\chi^{\dagger \prime}$ at $z=z_{\mathrm{c}}$. The solid line indicates $0.058 L^{2.37}$. Figure 3 shows the size dependence of $U$ defined by (6) at $z=z_{\mathrm{c}}$. We cannot extract a reliable estimate in $L=+\infty$ since we do not know the correction to scaling of this system. It will have a value between 0.54 and $2 / 3$. 


\section{Summary}

We carry out the Monte Carlo simulations of the hard-sphere lattice gas on the simple cubic lattice with nearest neighbour exclusion under fully periodic boundary conditions. Using the finite-size scaling, we obtain the critical exponents, $\beta / \nu=0.313(9)$ and $\gamma / \nu=2.37(2)$. They satisfy the scaling relation, $2 \beta+\gamma=d \nu$.

The critical exponents of the three-dimensional Ising model have been estimated, $\beta / \nu=0.518(7)$ and $\gamma / \nu=1.9828(57)$ [32]. These values do not agree with our results of the hard-sphere lattice gas. The value of the fourthorder cumulant of the Ising model on the simple cubic lattice under fully periodic boudary conditions is as follows. 0.44(2) [20], 0.4677(23) [33], and $0.4637(13)$ [34. We see that these values are smaller than those of the hardsphere lattice gas as seen in figure 3. While there is evidence that the hardsphere lattice gas on the square lattice belongs to the Ising universality class in two dimensions as described in section 1, it does not seem that the hardsphere lattice gas on the simple cubic lattice falls into the universality class of the three-dimensional Ising model.

\section{Acknowledgements}

We have carried out the simulations on the HITAC S-3600 computer under the Institute of Statistical Mathematics Cooperative Research Program (94ISM.CRP-43 and 95-ISM-CRP-37) and on two personal computers with the 486DX2/66MHz CPU and the Linux operating system (SLS 1.03 + JE 0.9.3, Slackware 2.0.0 + JE 0.9.5). This study was supported by a Grant-in-Aid for Scientific Research from the Ministry of Education, Science and Culture, Japan. 


\section{References}

[1] C. Domb, Nuovo Cimento 9 Suppl. (1958) 9.

[2] H.N.V. Temperley, Proc. Phys. Soc. 74 (1959) 183.

[3] D.M. Burley, Proc. Phys. Soc. 75 (1960) 262.

[4] D.S. Gaunt and M.E. Fisher, J. Chem. Phys. 43 (1965) 2840.

[5] D.S. Gaunt, J. Chem. Phys. 46 (1967) 3237.

[6] G. Kamieniarz and H.W.J. Blöte, J. Phys. A 26 (1993) 6679.

[7] D.M. Burley, Proc. Phys. Soc. 77 (1961) 451.

[8] L.K. Runnels, Phys. Rev. Lett. 15 (1965) 581.

[9] L.K. Runnels and L.L. Combs, J. Chem. Phys. 45 (1966) 2482.

[10] F.H. Ree and D.A. Chesnut, J. Chem. Phys. 45 (1966) 3983.

[11] R.J. Baxter, I.G. Enting, and S.K. Tsang, J. Stat. Phys. 22 (1980) 465.

[12] R.J. Baxter, J. Phys. A 13 (1980) L61.

[13] H. Meirovitch, J. Stat. Phys. 30 (1983) 681.

[14] A. Yamagata, Physica A 215 (1995) 511.

[15] H.W.J. Blöte and X.-N. Wu, J. Phys. A 23 (1990) L627.

[16] D.P. Landau, Phys. Rev. B 16 (1977) 4164.

[17] Z. Ràcz, Phys. Rev. B 21 (1980) 4012.

[18] R.J. Baxter and P.A. Pearce, J. Phys. A 15 (1982) 897.

[19] G. Kamieniarz and H.W.J. Blöte, J. Phys. A 26 (1993) 201.

[20] K. Binder, Z. Phys. B 43 (1981) 119.

[21] A.D. Bruce, J. Phys. A 18 (1985) L873. 
[22] D. Nicolaides and A.D. Bruce, J. Phys. A 21 (1988) 233.

[23] F.Y. Wu, Rev. Mod. Phys. 54 (1982) 235; 55 (1983) 315 (erratum).

[24] K. Binder, Monte Carlo Methods in Statistical Physics, K. Binder, ed. (Springer, Berlin, 1979) p. 1.

[25] K. Binder and D. Stauffer, Applications of the Monte Carlo Method in Statistical Physics, 2nd Ed., K. Binder, ed. (Springer, Berlin, 1987) p. 1.

[26] N. Ito and Y. Kanada, Supercomputer 5 (1988) 31.

[27] N. Ito and Y. Kanada, Proceedings of Supercomputing '90 (IEEE Computer Society Press, Los Alamitos, 1990) p. 753.

[28] M.E. Fisher, Critical Phenomena, Proc. 1970 Enrico Fermi Summer School, Vol. 51, M.S. Green, ed. (Academic Press, New York, 1970) p. 1.

[29] M.N. Barber, Phase Transitions and Critical Phenomena, Vol. 8, C. Domb and J.L. Lebowitz, eds. (Academic Press, London, 1983) p. 145 .

[30] V. Privman, Finite Size Scaling and Numerical Simulation of Statistical Systems, V. Privman, ed. (World Scientific, Singapore, 1990) p. 1.

[31] W.H. Press, S.A. Teukolsky, W.T. Vetterling, and B.P. Flannery, Numerical Recipes in C: The Art of Scientific Computing, 2nd Ed. (Cambridge University Press, Cambridge, 1992) Chapters 6 and 15.

[32] A.M. Ferrenberg and D.P. Landau, Phys. Rev. B 44 (1991) 5081.

[33] P.-Y. Lai and K.K. Mon, Phys. Rev. B 40 (1989) 11120.

[34] H.W.J. Blöte and G. Kamieniarz, Physica A 196 (1993) 445. 


\section{Figure captions}

Figure 1 Size dependence of the staggered density, $m^{\dagger \prime}$, defined by (3) at $z=z_{\mathrm{c}}$. The solid line indicates $0.48 L^{-0.313}$. Errors are less than the symbol size.

Figure 2 Size dependence of the staggered compressibility, $\chi^{\dagger \prime}$, defined by (5) at $z=z_{\mathrm{c}}$. The solid line indicates $0.058 L^{2.37}$. Errors are less than the symbol size.

Figure 3 Size dependence of the fourth-order cumulant, $U$, defined by (6) at $z=z_{\mathrm{c}}$. 


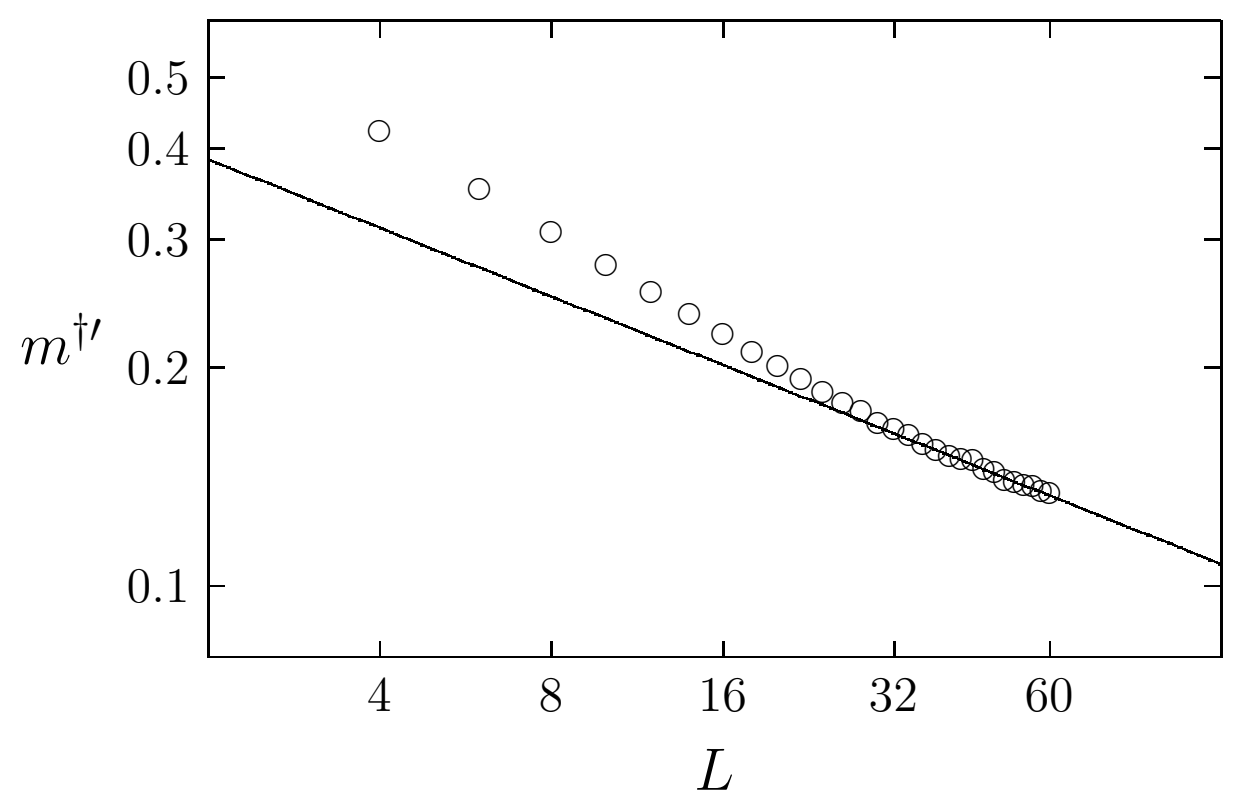

Figure 1

Atsushi Yamagata 


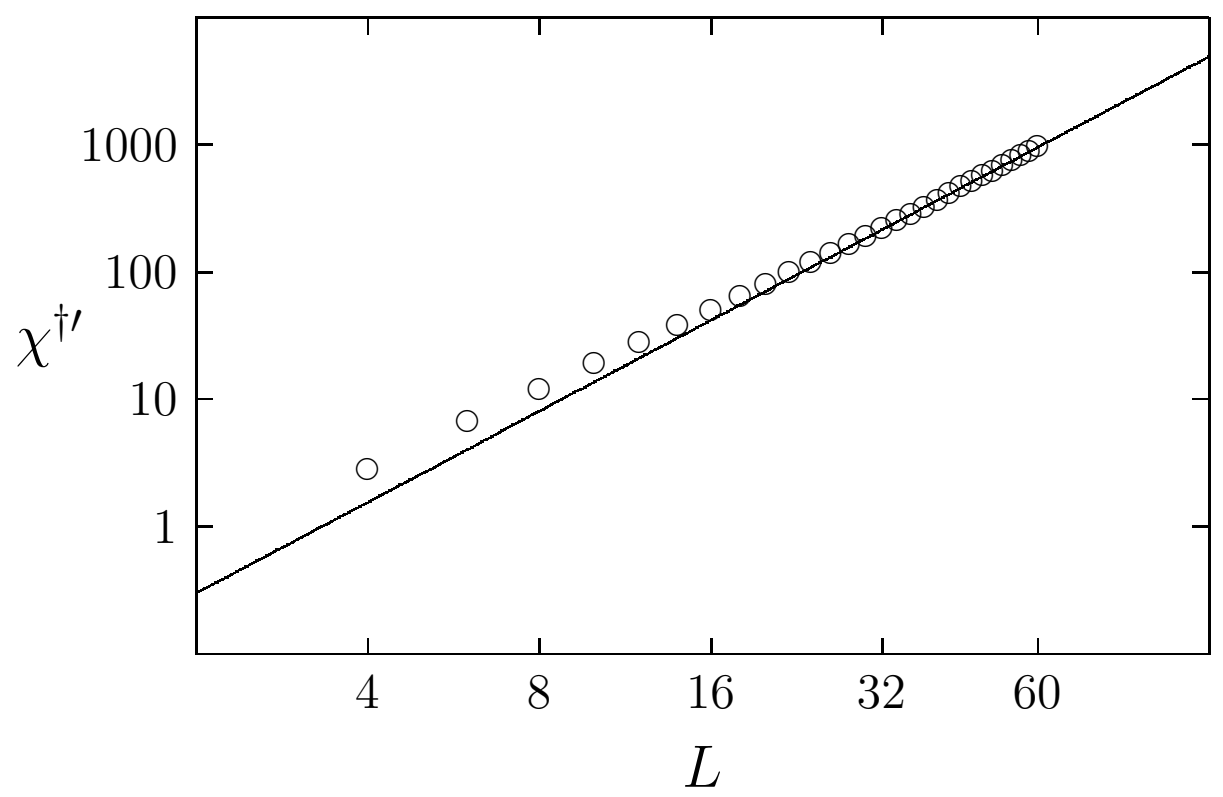

Figure 2

Atsushi Yamagata 


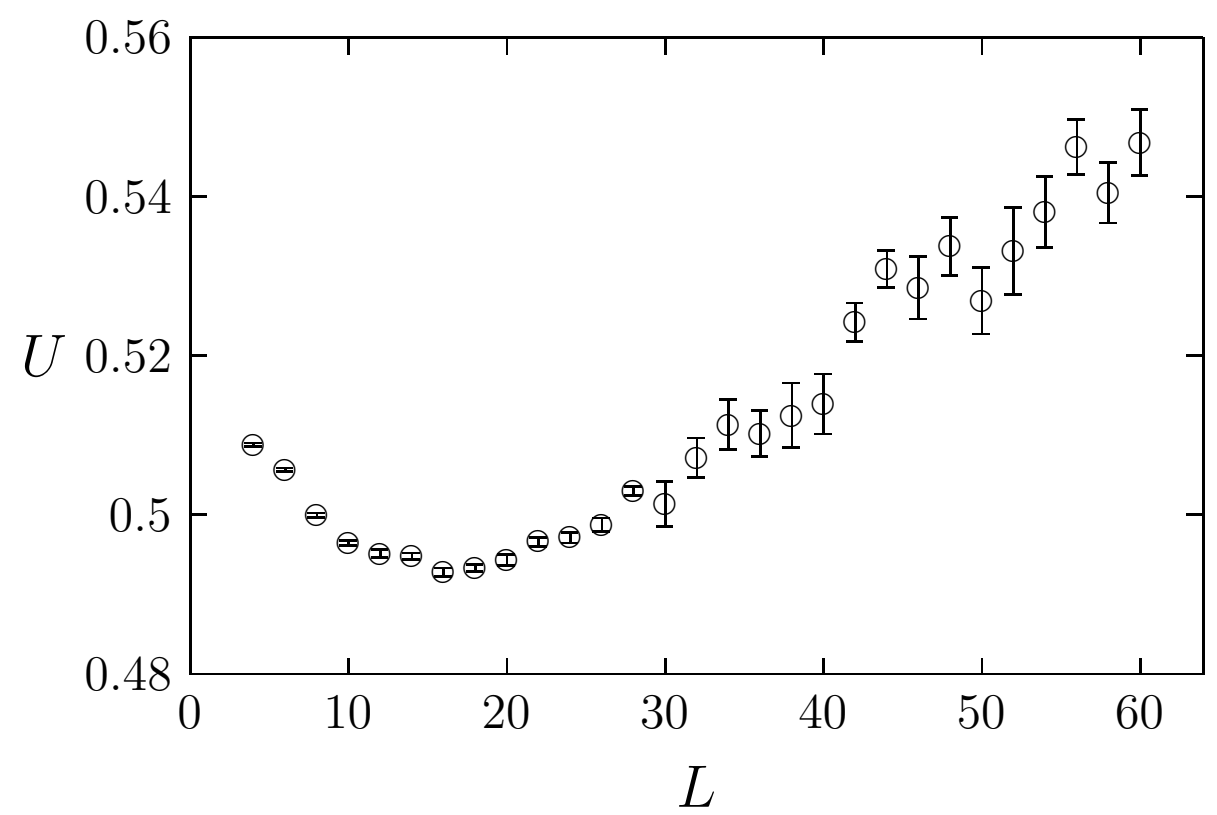

Figure 3

Atsushi Yamagata 